\title{
Patientenmanagement bei fazialer Umstellungsosteotomie
}

\author{
Astrid Toferer und Bernhard Taxer
}

Eine faziale Umstellungsosteotomie optimiert neben der Okklusion und Funktion auch die Ästhetik. Für den positiven Heilungsverlauf sind ein multimodaler Ansatz, gelebte Interdisziplinarität und ein erfolgreiches Schmerzmanagement unerlässlich. Die Geschichte von Lisa D. zeigt das peri- und postoperative Management aus multiprofessioneller Sicht und beleuchtet langfristige Herausforderungen.

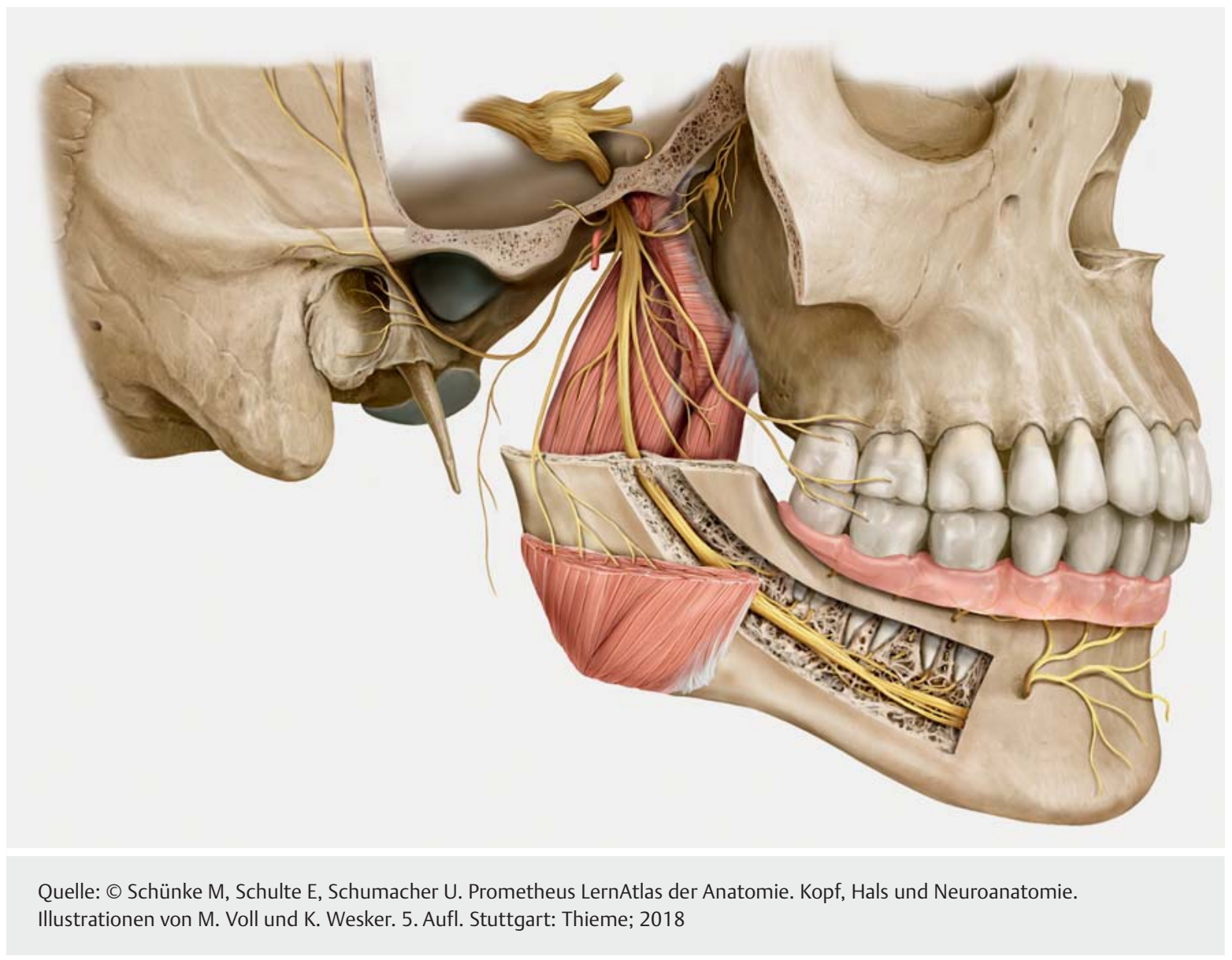

Orofaziale Schmerzen umfassen ein breites Spektrum verschiedener Erkrankungen [1]. Ihre Zuordnung und Behandlung ist meist komplex und erfordert stets das Berücksichtigen biopsychosozialer Komponenten [2]. Neben einer empathischen Kommunikationsstrategie sind ein interprofessionelles perioperatives Management bei der akuten Schmerzversorgung sowie die Absprache mit Logopäden und Physiotherapeuten notwendig [3,4]. Auch beim präoperativen Screening auf Risikofaktoren ist der biopsychosoziale Ansatz elementar [5]. 


\section{Rolle der Physiotherapie}

Bei fazialen Umstellungsosteotomien spielen Physiotherapeuten und Logopäden eine große Rolle. Neben der Sprech- und Zungenfunktion liegt deren Fokus auf Parafunktionen wie Schlaf-oder Wach-Bruxismus, pathologischen Zungenpositionen oder oralen Tics. Eine frühzeitige Behandlung soll postoperative Rezidive wie bspw. einen frontoffenen Biss verhindern.

Präoperatives Management Weil eine Umstellungsosteotomie die Muskel- und Gelenkposition im Raum geringgradig verändert, kann ein Patient postoperativ anfangs Schwierigkeiten haben, sich an seine neue Bisssituation zu gewöhnen [6]. Auch kraniomandibuläre Dysfunktionen wie Zwangsbissführungen, muskuläre Dysbalancen und Haltungsdefizite v.a. der Halswirbelsäule (HWS) erschweren die Eingewöhnung, verhindern die physiologische Gelenkposition für die OP-Planung und belasten den dorsokranialen Belastungsvektor der suprahyoidalen Muskulatur [6,7]. Folglich soll das kraniomandibuläre System präoperativ durch orofazial spezialisierte Physiotherapie, Aufbiss-Schienen, ggf. Verhaltenstherapie und Muskelrelaxantien ins Gleichgewicht gebracht werden. Trotz ,Conflicting evidence‘ zwischen präoperativem Management und sofortiger chirurgischer Intervention wird so die postoperative Umgewöhnung unterstützt [8].

Postoperatives Management Für den Heilungsprozess ist die postoperative Schmerztherapie elementar und verhindert eine Schmerzchronifizierung. Die Physiotherapie soll den postoperativen Verlauf positiv beeinflussen [4]. Schmerzhafte ödematöse Schwellungen werden durch Hochlagern des Oberkörpers und konsequente Kryotherapie reduziert. Angesichts mangelhafter Evidenz kann die Manuelle Lymphdrainage nicht grundlegend empfohlen werden; im klinischen Alltag wird diese jedoch regelmäßig angewandt und von den Patienten zumeist als wohltuend empfunden.

\section{Fallbeispiel}

Auf Grund eines ausgeprägten Distalbisses war bei Lisa D. eine chirurgisch-kieferorthopädische Therapie indiziert. Die damals 21-jährige Fabrikarbeiterin litt unter einer eingeschränkten Kaufunktion und empfand ihr stark fliehendes Kinn ästhetisch und funktionell als störend. Für die leidenschaftliche Go-Kart-Fahrerin und Mountainbikerin war es schwierig, einen Helm zu fixieren.

\section{ZUSATZINFO}

\section{Dysgnathie-Chirurgie}

Als Dysgnathien werden ausgeprägte Fehlstellungen der Zähne oder Kiefer bezeichnet. Die konservative Therapie führt bei ausgeprägten Fällen häufig nicht zum gewünschten Behandlungsergebnis [7]. Die chirurgische Kieferorthopädie korrigiert skelettale Lageanomalien im Gesichtsschädel. Über eine Umstellungsosteotomie wird eine harmonische, schädelbezogene Einstellung der Kieferbasen zueinander und eine korrekte Okklusion des Kausystems erreicht, um eine gleichmäßige Belastung von Zähnen und Zahnhalteapparat sowie ein harmonisches Gesichtsprofil zu gewährleisten [9].

\section{Anamnese}

Komorbiditäten In der Anamnese berichtete die Patientin von einer Leukämie im Kindesalter und Bestrahlungen im Kopfbereich. Regelmäßige Kontrolluntersuchungen waren unauffällig. Vor etwa zehn Jahren erlitt sie beim Go-Kart-Sport ein Schleudertrauma (WhiplashAssociated Disorder, WAD), das sich in rezidivierenden Kopf- und Nackenschmerzen äußerte.

\section{Umstellungsosteotomie}

Nach ausführlicher Therapieplanung wurde eine Umstellungsosteotomie des Ober- und Unterkiefers mit Kinnkorrektur festgelegt. Präoperativ wurden zwei Prämolaren im Unterkiefer gezogen und die Zahnbögen mit Brackets ausgeformt. Eineinhalb Jahre später wurde die OP komplikationslos durchgeführt ( $\triangleright$ Abb. 1).

\section{Postoperative stationäre Phase}

Trotz üblicher fazialer Schwellung, erschwerter Atmung und der Einnahme flüssiger Kost blieb die Patientin entspannt. Neben einer Antibiose erhielt sie eine analgetische und antiphlogistische Therapie. Als Maximalschmerz gab sie 2/10 auf der Numerischen-Rating-Skala (NRS) an. Physiotherapeutisch wurde sie mit Lymphdrainagen und sanften Massagen zur Ödem- und Schmerzreduktion versorgt.

Dysästhesie Am fünften Tag imponierte eine Dysästhesie der Nn. mentali im Bereich von Kinn und Unterlippe. Die Sensibilitätsstörung war angesichts von Schwellung und intraoperativer Manipulation nicht ungewöhnlich. Für die nervale Regeneration wurde ein Vitamin-B1-B6-Präparat rezeptiert. Vier Wochen später besserte sich die Dysästhesie. Lisa D. war mit dem postoperativen Verlauf und ihrem Erscheinungsbild sehr zufrieden ( $\triangleright$ Abb. 2). 

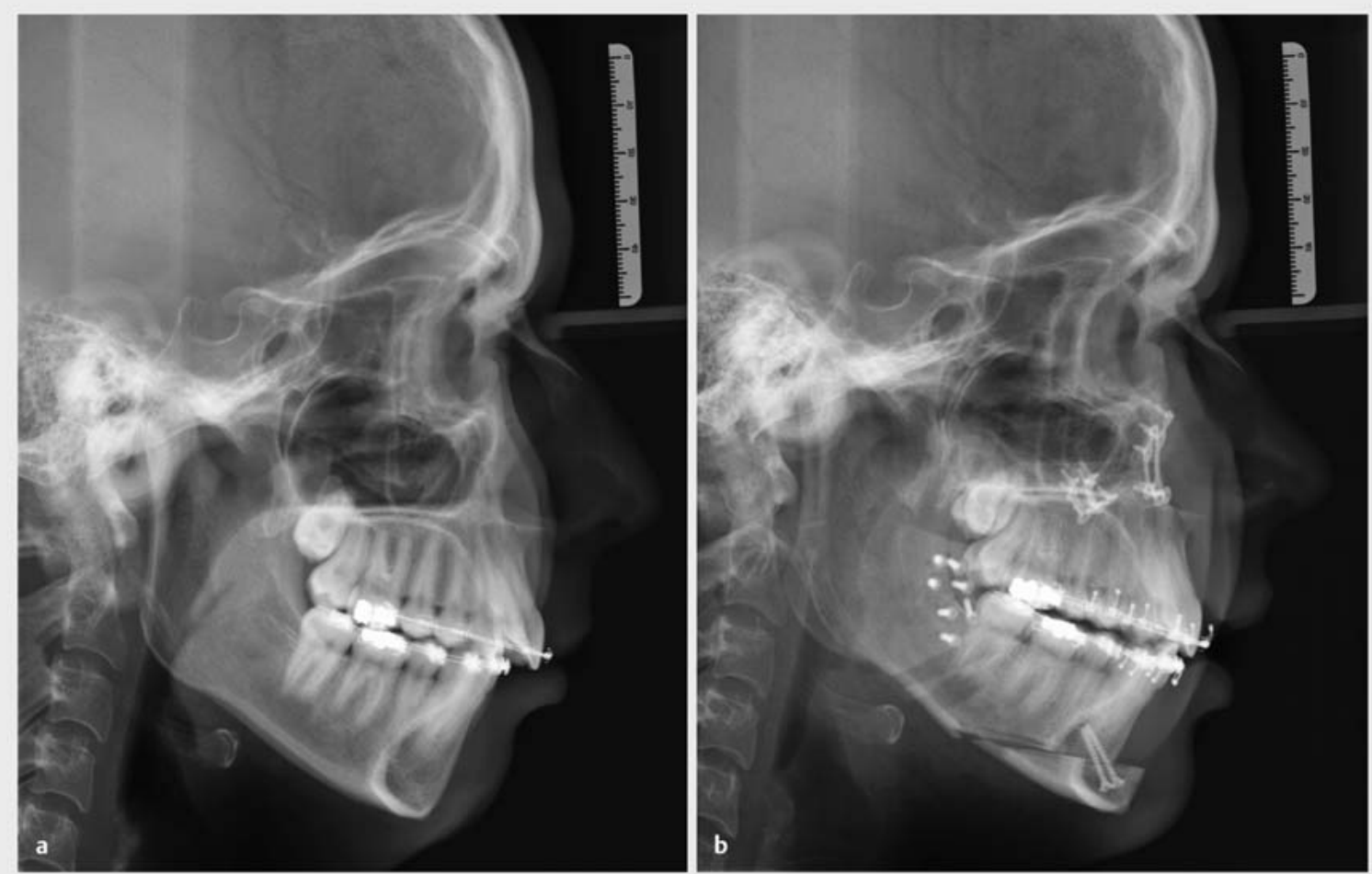

- Abb. 1 a Präoperatives Röntgenbild. b Postoperatives Röntgenbild. Das Kinn wurde um 5 mm vorverlagert. Quelle: A. Toferer

\section{Postoperative ambulante Phase}

In der ambulanten Nachkontrolle berichtete die Patientin erstmals über eine ziehende Schmerzsensation im linken Gaumen auf Höhe der ersten beiden Molaren. Bei der Untersuchung zeigte sich ein retinierter Weisheitszahn, dessen Wurzel fast bis zum Maximalpunkt des Schmerzes in den Gaumen ragte.

\section{Exazerbation der Beschwerden}

Angesichts der ziehenden Schmerzen wurden der Weisheitszahn und das Osteosynthese-Material im Oberkiefer entfernt. Postoperativ verstärkten sich hingegen die Oberkieferschmerzen (8/10 NRS) und wurden jetzt von einer Druckhyperalgesie begleitet. Die Dysästhesie des linken N. mentalis trat erneut auf. Die Patientin beschrieb „stechende“ Schmerzen (4/10 NRS).

\section{Erklärung der Schmerzzunahme}

Neuropathie Bei Umstellungsosteotomien können sensible Nervenbahnen verletzt werden. Im Oberkiefer sind v.a. die sensible Versorgung von Zähnen und Schleimhaut betroffen, im Unterkiefer v.a. der N. mandibularis, was mit Sensibilitätsstörungen im Unterlippen- und Kinnbereich imponiert [9]. Zumeist kommt es zur Restitution der Beschwerden. Bleibende Nervenbeeinträchtigungen beschränken sich in der Regel auf kleinere Areale. Nur selten kommt es - wie im geschil- derten Fall - zu neuropathischen Schmerzsensationen und Allodynien.

Folgen Da die Empfindlichkeit des nozizeptiven Systems nach einer Gewebeschädigung durch periphere und zentrale Sensibilisierung bereits gesteigert sein kann, birgt jeder weitere Eingriff die Gefahr einer Schmerzchronifizierung [10-14]. Trotz mangelhafter Studienlage zur postoperativen Schmerzversorgung in der Kieferchirurgie sollten schmerzunterhaltende Noxen beseitigt und jede weitere invasive Maßnahme gut abgewogen werden $[3,4]$.

\section{Multiprofessionelle Therapie}

Die persistierende Neuropathie und Allodynie deuteten auf eine drohende Schmerzchronifizierung hin [15]. Demgemäß wurde ein multiprofessionelles Team involviert und der Fall jetzt auf biopsychosozialer Ebene betrachtet. Die im Anschluss dargestellten Maßnahmen wurden in der angeführten Reihenfolge bzw. Progression angewandt. 


\section{Medikamentöse Therapie}

Auf Grund der Schmerzzunahme und Taubheit im linken Kinnbereich wurde die Patientin zunächst medikamentös versorgt. Bei neuropathischen Schmerzen werden Antikonvulsiva, trizyklische Antidepressiva, selektive Serotonin- und Noradrenalin-Wiederaufnahme-Hemmer und Opioid-Analgetika eingesetzt [15-19]. Bei der Patientin stellten sich jedoch nur Schwindelgefühle ein. Auch eine opioid-haltige Mundspülung und ein systemisch verabreichtes Opioid brachten keine Besserung [20].

\section{Invasive Therapieversuche}

Nervenblockaden

Nervenblockaden dienen als Diagnostikum und als Therapeutikum. Mittels zweier diagnostischer Infiltrationen wurden die sensiblen Einzugsgebiete der linken Nn. mentalis und palatinus major als neuropathische Areale detektiert. Anschließend wurde dort ein Infiltrationszyklus mit einem langwirkenden Lokalanästhetikum initiiert. Die analgetische Wirkung hielt rund drei Wochen an; danach imponierte trotz weiterer Infiltrationen die gleiche Schmerzintensität wie zuvor.

\section{Neurochirurgie}

Weil die Patientin unter einer Trigeminusneuropathie litt, schieden eine Thermokoagulation im Bereich des Ganglion Gasseri oder eine Dekompressions-OP nach Jannetta als Behandlungsoptionen aus. Diese Interventionen werden nur bei Trigeminusneuralgien mit einer mikrovaskulär bedingten Kompression im Bereich der Nerveneintrittszone empfohlen [21].

\section{GLOA}

Bei einer ganglionären lokalen Opioidanalgesie (GLOA) können sympathisch unterhaltene Schmerzsyndrome durch Opioid-Infiltration des Ganglion cervicale superius ausgeschaltet werden [22]. Die Punktion in die Rachenhinterwand war bei der Patientin aufgrund ihres starken Würgereizes nicht möglich.

Dieser letzte erfolglose invasive Therapieversuch wurde parallel zur Physiotherapie durchgeführt. Der Fokus wurde nun auf physiotherapeutische und psychosoziale Aspekte gelegt.

\section{Physiotherapeutisches Assessment}

\section{Anamnese}

Rund ein Jahr nach ihrer ersten OP erschien die Patientin zur spezialisierten orofazialen Physiotherapie. Als Hauptproblem reklamierte sie ihre massiven Gaumenschmerzen und das Taubheitsgefühl der linken Kinnregion. Zusätzlich gab sie rezidivierende, linksseitige Kopf- und Nackenschmerzen an, die u.a. mit ihrem WAD in Zusammenhang standen ( $\triangleright$ Abb. $\mathbf{3}$ ).
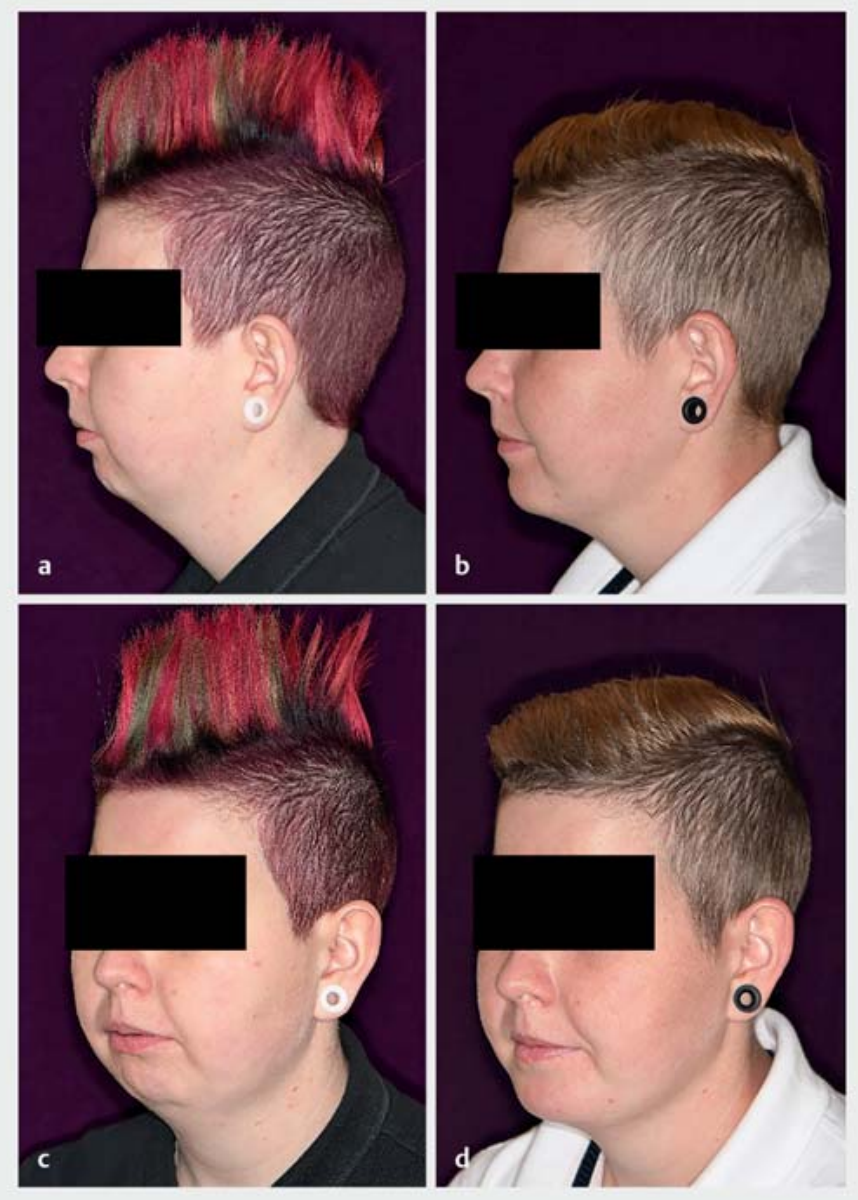

- Abb. 2 a Profilbild präoperativ. b Profilbild postoperativ. c Frontalansicht präoperativ. d Frontalansicht postoperativ. Quelle: A. Toferer.

\section{Schmerzanamnese zum Bodychart}

- 5 D nach Coman (Dizziness, Diplopia, Drop Attacks, Dysphagia, Dysarthria): negativ

- $3 \mathrm{~N}$ (Nausea, Nystagmus, Numbness): negativ bis auf das Taubheitsgefühl in Symptom 1'

- 1 A (Ataxie): negativ

- Schmerzverhalten:

- die Symptome 1 und 1' bestanden unabhängig von mechanischen oder thermischen Einflüssen, es konnten keine Faktoren für eine Verschlechterung/ Verbesserung angegeben werden

- 24 Stunden-Verhalten: die Patientin spürte die Symptome 1 und 1' bereits nach dem Aufstehen und dann konstant über den ganzen Tag; kein Nachtschmerz

\section{Neurologische Untersuchung}

Eine neurologische Untersuchung mit Fokus auf die $\mathrm{Nn}$. facialis und trigeminus offenbarte sensorische Beeinträchtigungen und einen münzgroßen Sensibilitätsausfall an der Austrittsstelle des N. mentalis. 
1: Gaumenseite links intraoral ziehend, konstant, tief,

massiv berührungsempfindlich

NRS 7-8/10

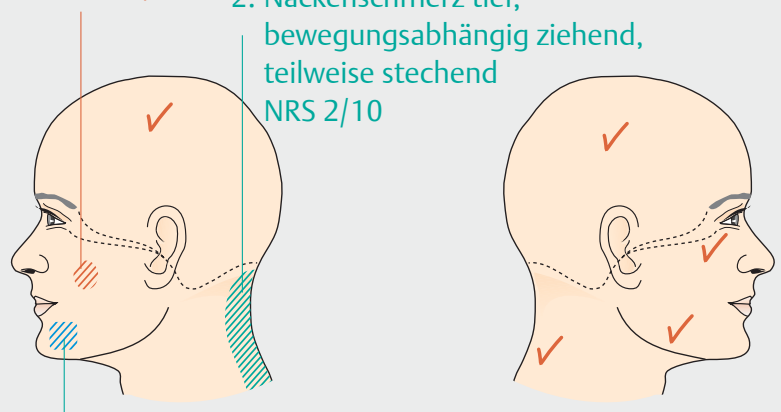

$1^{`}$ : Anästhesie trotzdem teilweise auch schmerzhaft, drückend, stechend, konstant, oberflächlich NRS $1-4 / 10$

- Abb. 3 Bodychart der Patientin ein Jahr nach der Umstellungsosteotomie. Quelle: B. Taxer; graf. Umsetzung: Thieme Gruppe.

\section{LANSS}

Im ,Leeds Assessment of Neuropathic Signs and Symptoms، (LANSS) gab die Patientin Gefühlsstörungen und Berührungsempfindlichkeiten sowie „anfallsartige“ und „brennende“ Schmerzsensationen an. Zudem fühlte sich das Schmerzareal „unangenehm“, „empfindlicher“ und „taub“ an, wenn sie mit einem Finger darüber rieb. Der LANSS-Score betrug 19 von 24 Punkten und bestätigte den Verdacht auf ein neuropathisches Geschehen.

\section{Funktionelle Untersuchung}

Kiefergelenk extraoral

Die funktionelle Untersuchung zeigte eine Mundöffnung von $4,9 \mathrm{~cm}$ mit einem dezenten Schwingungsmuster nach links ohne einseitige Deflexion. Die Laterotrusion betrug je $1 \mathrm{~cm}$ und fühlte sich für Lisa $\mathrm{D}$. rechts steifer und unangenehmer an.

\section{Kiefergelenk intraoral}

Die intraorale Untersuchung lieferte Hinweise auf eine linksseitig eingeschränkte arthrogene Komponente v.a. bei der Zusatzbewegung nach transversal lateral. Auch hier reklamierte die Patientin ein unangenehm steifes Gefühl. Die palpierbaren Kaumuskeln reagierten links zwar drucksensibler, die Symptomatik konnte jedoch nicht provoziert werden. Die intraorale Inspektion war unauffällig.

Interpretation Die klinischen Zeichen ließen zwar auf eine arthrogene Komponente schließen, wurden aber für das Hauptproblem eher als nozizeptiv beitragende On-Off-Mechanismen interpretiert [23]. Diese Zeichen wurden beim weiteren Clinical Reasoning berücksichtigt. Die muskuläre Sensibilität wurde als ,Referred Pain' bewertet und nicht als myogene Temporomandibular Disorder (TMD) auf Grund der beschriebenen Funktionalität. Weil auch die erhöhte Mechanosensitivität zu einem veränderten Bewegungsverhalten und der beschriebenen Symptomatik beitragen kann, wurde nun auch die Neurodynamik der betroffenen Nervenäste geprüft [24, 25].

HWS

Nicht zuletzt auf Grund des zehn Jahre zurückliegenden WADs wurde die HWS manualtherapeutisch untersucht. Es zeigte sich eine dominant hochzervikal eingeschränkte aktive Flexionsbewegung begleitet von einem Ziehen bis in die BWS. Die aktive HWS-Rotation war mit je $90^{\circ}$ normal; die Rechtsrotation löste die hochzervikale Symptomatik aus ( $\mathbf{A b b} . \mathbf{3}$ ).

Der Flexions-Rotations-Test diente als Screening für C0C2 und war links um ca. $15^{\circ}$ eingeschränkt. Auch er triggerte die hochzervikale Symptomatik. Passive Zusatzbewegungen bestätigten die erhöhte Mechanosensitivität und hochzervikale Stiffness.

Interpretation Im kraniozervikalen Bereich war ebenfalls von einer dominant nozizeptiven On-Off-Komponente auszugehen. Weil es sich um aufrechterhaltende periphere Strukturen handelte, wurde die HWS fortan mitberücksichtigt und -behandelt.

\section{Physiotherapeutisches Management und Behandlung}

\section{Auswertung des Assessments}

Die Untersuchung ergab folgende Befunde:

- deutliche klinische Zeichen eines neuropathischen Schmerzmusters auf Grund des klinischen Bildes $(\triangleright$ Abb. 3 ) und des LANSS-Scores

- nicht kausale arthrogene TMD als beitragender Faktor der trigeminalen Sensibilisierung

- weitere beitragende kraniozervikale Faktoren auf Grund des WAD in der Krankengeschichte

\section{Therapieplan}

Anhand des Assessments wurden diverse Interventionen festgelegt.

Hands-On-Interventionen:

- Manuelle Therapie im Bereich der kraniomandibulären Region (muskulär, arthrogen, neural) [26-28]

- Manuelle Therapie der HWS

Hands-Off-Interventionen:

- Edukation [29]

- Trainingstherapie zur Grundlagenausdauer [10, 30, 31] 
Die Patientin wurde manualtherapeutisch extra- und intraoral mit „Gaumentechniken“ zur Desensibilisierung behandelt. Neurodynamische Techniken v.a. für die Trigeminusäste sorgten für kurzfristige Verbesserungen. Hierzu wurden der Patientin auch Heimübungen instruiert, die sie allerdings nur inkonsequent umsetzte. Dementgegen steigerte sie ihre Grundlagenausdauer auf Ergometer und Mountainbike.

\section{Fazit und Ausblick}

Ein operativer Eingriff steigert die Empfindlichkeit des nozizeptiven Systems. Daneben können sich zusätzliche Faktoren im Heilungsverlauf negativ auf eine trigeminale und extratrigeminale Sensibilisierung auswirken [32,33]. Im vorliegenden Fall spielten die klinischen Zeichen der kraniomandibulären und kraniozervikalen Region eine wesentliche Rolle zur Unterstützung dieser Hypothese.

Aus der subjektiven Untersuchung ergab sich die Überlegung, dass die Bestrahlung des Kopfbereichs im Kindesalter und das WAD schmerzunterhaltende Faktoren darstellen könnten. Aus psychosozialer Sicht stellte der Beruf als Fabrikarbeiterin mit regelmäßigen Nachtschichten und teils schwerer körperlicher und monotoner Arbeit einen weiteren Faktor dar.

Psychosoziale Aspekte Diverse Studien zeigen, dass sich Eingriffe in das orthognate System deutlich auf Persönlichkeit, Lebensqualität und das psychologische Profil der Betroffenen auswirken können [34-37]. Die ästhetischen Folgen einer OP und die damit verbundenen Herausforderungen auf der Beziehungsebene eines Patienten sind differenziert zu betrachten [37-40]. Diese psychosozialen Determinanten müssen valide überprüft und in einer multimodalen Behandlung berücksichtigt werden, ansonsten bleiben sie reine Spekulation.

Herausforderung Ein optimales Therapiemanagement detektiert frühzeitig alle schmerzunterhaltenden biopsychosozialen Faktoren und verhindert so eine Schmerzchronifizierung. Mittel der Wahl sind routinemäßige Untersuchungen und Fragebogen. Geeignet sind Questionnaires zur Lebensqualität, craniofazial-spezifische Assessments, Fragebogen zu Alexithymie, Katastrophisierung, psychosozialen Risikofaktoren und zur Schmerzvalidation [41-45].

Im Sinne eines umfassenden Clinical Reasonings für alle beteiligten Berufsgruppen macht es Sinn, mittels Qualitätszirkeln einheitliche Verfahren zu wählen, sodass Behandler und Betroffene bestmöglich profitieren.

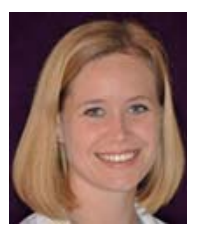

Dr. Dr. Astrid Toferer ist Assistenzärztin für Mund-, Kiefer- und Gesichtschirurgie an der Universitätsklinik Graz. Ihr Interessensschwerpunkt sind Kiefergelenkserkrankungen und faziale Schmerzsyndrome. In diesem Rahmen befindet sie sich aktuell sowohl in der Ausbildung für das ÖÄK-Diplom „Spezielle Schmerztherapie" als auch im Curriculum „Funktion, Dysfunktion, CMD und Schmerz“ der ,Akademie Praxis und Wissenschaft ${ }^{*}$ (APW). Zusätzlich absolviert sie die Doctoral School „Dentistry and Maxillofacial Surgery“ (scient.med.) an der medizinischen Universität Graz und ist als Zahnärztin in einer Ordination tätig.

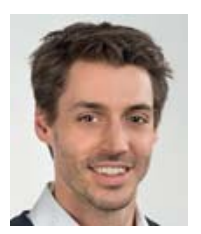

Bernhard Taxer (MSc PT, OMT) ist freiberuflicher Physiotherapeut und als Dozent für den Studiengang Physiotherapie an der FH JOANNEUM in Graz tätig. Sein Interessensschwerpunkt umfasst Clinical Reasoning bei neurologisch-muskuloskelettalen Beschwerden, persistierende Schmerzsyndrome mit dem Fokus auf Neuropathie sowie kraniofaziale Krankheitsbilder. Diesen Interessenfeldern geht er als NOI-Instruktor und Lehrassistent im CRAFTA ${ }^{\circledR}$-Konzept nach. Aktuell befindet er sich in einem PhDProgramm (scient.med.) an der Paracelsus Medizinischen Universität Salzburg.

Korrespondenzadresse

Dr. med. univ. Dr. med. dent. Astrid Toferer

Medizinische Universität Graz

Klinische Abteilung für Mund-, Kiefer- und Gesichtschirurgie Auenbruggerplatz 5/6

8036 Graz

Österreich

astrid.toferer@medunigraz.at

Literatur

[1] International Headache Society. International Classification of Orofacial Pain, 1st edition (ICOP). Cephalalgia 2020; 40: 129221. doi: $10.1177 / 0333102419893823$

[2] Katon W, Kleinman A. A Biopsychosocial Approach to Surgical Evaluation and Outcome. West J Med 1980; 133: 9-14

[3] Rabbitts JA, Kain Z. Perioperative Care for Adolescents Undergoing Major Surgery: A Biopsychosocial Conceptual Framework. Anesth Analg 2019; 129: 1181-1184. doi:10.1213/ ANE. 0000000000004048

[4] Likar R, Jaksch W, Aigmüller T et al. Interdisziplinäres Positionspapier „Perioperatives Schmerzmanagement“. Schmerz 2017; 31: 463-482. doi:10.1007/s00482-017-0217-y

[5] Penlington C, Ohrbach R. Biopsychosocial assessment and management of persistent orofacial pain. Oral Surg 2020; 13 : 349-357. doi:10.1111/ors.12470 
[6] Stelzenmüller W, Wiesner ]. Therapie von Kiefergelenkschmerzen: Ein Behandlungskonzept für Zahnärzte, Kieferorthopäden und Physiotherapeuten. 2., überarbeitete und erweiterte Edition. Stuttgart: Thieme; 2010

[7] Schwenzer N, Ehrenfeld M. Mund-Kiefer-Gesichtschirurgie. 4., vollständig überarbeitete und erweiterte Edition. Stuttgart: Thieme; 2010

[8] Peiró-Guijarro MA, Guijarro-Martínez R, Hernández-Alfaro F. Surgery first in orthognathic surgery: A systematic review of the literature. Am J Orthod Dentofacial Orthop 2016; 149: 448-462. doi:10.1016/j.ajodo.2015.09.022

[9] Hausamen J-E, Machtens E, Reuther JF, et al. Mund-, Kieferund Gesichtschirurgie. 4. Aufl. Berlin Heidelberg: SpringerVerlag; 2012. doi:10.1007/978-3-642-17801-6

[10] Jesson T, Runge N, Schmid AB. Physiotherapy for people with painful peripheral neuropathies: a narrative review of its efficacy and safety. PAIN Rep 2020; 5: 1-e834. doi:10.1097| PR9.0000000000000834

[11] Adams LM, Turk DC. Central sensitization and the biopsychosocial approach to understanding pain. J Appl Biobehav Res 2018; 23 (2): e12125. doi:10.1111/jabr.12125

[12] Woolf C]. Central sensitization: Implications for the diagnosis and treatment of pain. Pain 2011; 152: S2-S15. doi:10.1016/ j.pain.2010.09.030

[13] van Griensven H, Schmid A, Trendafilova T, et al. Central Sensitization in Musculoskeletal Pain: Lost in Translation? J Orthop Sports Phys Ther 2020; 50: 592-596. doi:10.2519/jospt. 2020.0610

[14] Murnion BP. Neuropathic pain: current definition and review of drug treatment. Aust Prescr 2018; 41: 60-63. doi:10.18773/ austprescr.2018.022

[15] Scholz ], Finnerup NB, Attal N, et al. The IASP classification of chronic pain for ICD-11: chronic neuropathic pain. PAIN 2019; 160: 53. doi:10.1097/j.pain.0000000000001365

[16] Beubler E. Kompendium der medikamentösen Schmerztherapie: Wirkungen, Nebenwirkungen und Kombinationsmöglichkeiten. 6. Aufl. Berlin Heidelberg: Springer-Verlag; 2016. doi:10.1007/978-3-662-48827-0

[17] Finnerup NB, Attal N, Haroutounian S, et al. Pharmacotherapy for neuropathic pain in adults: systematic review, meta-analysis and updated NeuPSIG recommendations. Lancet Neurol 2015; 14: 162-173. doi:10.1016/S1474-4422(14)70251-0

[18] Schlereth T, Birklein F, Wasner G, et al. Diagnose und nicht interventionelle Therapie neuropathischer Schmerzen, S2k-Leitlinie. 2019. Im Internet (Stand: 28.12.2020): https://dgn.org/wp-content/uploads/2013/01/030114_LL_ Neuropathische_Schmerzen_2019.pdf

[19] Colloca L, Ludman T, Bouhassira D, et al. Neuropathic pain. Nat Rev Dis Primer 2017; 3: 17002. doi:10.1038/nrdp.2017.2

[20] Witt E, Palla S. Mundbrennen. Schmerz 2002; 16: 389-394. doi:10.1007/s00482-002-0149-y

[21] Paulus W, Evers S, May A et al. Therapie und Prophylaxe von Gesichtsneuralgien und anderen Formen der Gesichtsschmerzen. Schmerz 2003; 17: 74-91. doi:10.1007/s00482-0020179-5

[22] Benrath ], Hatzenbühler M, Fresenius M et al. Repetitorium Schmerztherapie: Zur Vorbereitung auf die Prüfung „Spezielle Schmerztherapie“. 4. Aufl. 2015 Edition. Berlin, Heidelberg: Springer; 2015

[23] Schiffman E, Ohrbach R, Truelove E et al. Diagnostic Criteria for Temporomandibular Disorders (DC/TMD) for Clinical and Research Applications: Recommendations of the International
RDC/TMD Consortium Network and Orofacial Pain Special Interest Group. J Oral Facial Pain Headache 2014; 28: 6-27

[24] von Piekartz H. Kiefer, Gesichts- und Zervikalregion - Neuromuskuloskeletales Assessment und Behandlungsstrategien. 2. Aufl. Thieme Verlag; 2015

[25] von Piekartz H, Doppelhofer D. Gesichtsschmerzen und der neurodynamische Test des N. mandibularis. Physiotherapie 2007; 2. Im Internet (Stand: 29.12.2020): https://crafta.org/ artikel/art11.pdf

[26] Armijo-Olivo S, Pitance L, Singh V, et al. Effectiveness of Manual Therapy and Therapeutic Exercise for Temporomandibular Disorders: Systematic Review and Meta-Analysis. Phys Ther 2016; 96: 9-25. doi:10.2522/ptj.20140548

[27] Groß H. Einfluss Manueller Therapie an der oberen HWS auf die Schmerzempfindlichkeit der Kaumuskulatur bei CMD. manuelletherapie 2009; 13: 141-146. doi:10.1055/s-00281109719

[28] Knust M, von Piekartz H, Zalpour C. Wirkung von Manueller Therapie im Vergleich zu einem multimodalen Physiotherapieprogramm bei Patientinnen mit kraniomandibulärer Dysfunktion. physioscience 2007; 3: 109-116. doi:10.1055/s2007-963380

[29] Louw A, Diener I, Butler DS, et al. The Effect of Neuroscience Education on Pain, Disability, Anxiety, and Stress in Chronic Musculoskeletal Pain. Arch Phys Med Rehabil 2011; 92: 20412056. doi:10.1016/j.apmr.2011.07.198

[30] Almeida C, DeMaman A, Kusuda R, et al. Exercise therapy normalizes BDNF upregulation and glial hyperactivity in a mouse model of neuropathic pain: PAIN 2015; 156: 504-513. doi:10.1097/01.j.pain.0000460339.23976.12

[31] López-Álvarez VM, Modol L, Navarro X, et al. Early increasingintensity treadmill exercise reduces neuropathic pain by preventing nociceptor collateral sprouting and disruption of chloride cotransporters homeostasis after peripheral nerve injury: PAIN 2015; 156: 1812-1825. doi:10.1097/j.pain. 0000000000000268

[32] Park JW, Clark GT, Kim YK, et al. Analysis of thermal pain sensitivity and psychological profiles in different subgroups of TMD patients. Int J Oral Maxillofac Surg 2010; 39: 968-974. doi:10.1016/j.ijom.2010.06.003

[33] Fernández-de-las-Peñas C, Galán-del-Río F, Ortega-Santiago R, et al. Bilateral thermal hyperalgesia in trigeminal and extratrigeminal regions in patients with myofascial temporomandibular disorders. Exp Brain Res 2010; 202: 171-179. doi:10.1007/s00221-009-2121-x

[34] Cunningham S], Hunt NP, Feinmann C. Perceptions of outcome following orthognathic surgery. $\mathrm{Br}$ ] Oral Maxillofac Surg 1996; 34: 210-213. doi:10.1016/S0266-4356(96)90271-5

[35] Flanary CM, Barnwell GM, VanSickels JE, et al. Impact of orthognathic surgery on normal and abnormal personality dimensions: A 2-year follow-up study of 61 patients. Am J Orthod Dentofacial Orthop 1990; 98: 313-322. doi:10.1016/S0889-5406(05)81488-X

[36] Kiyak HA, Hohl T, West RA, et al. Psychologic changes in orthognathic surgery patients: A 24-month follow up. J Oral Maxillofac Surg 1984; 42: 506-512. doi:10.1016/02782391(84)90009-0

[37] Lee S, McGrath C, Samman N. Impact of Orthognathic Surgery on Quality of Life. J Oral Maxillofac Surg 2008; 66: 1194-1199. doi:10.1016/j.joms.2008.01.006

[38] Sinko K, Jagsch R, Benes B, et al. Facial aesthetics and the assignment of personality traits before and after orthognathic surgery. Int J Oral Maxillofac Surg 2012; 41: 469-476. doi:10.1016/j.ijom.2011.10.026 
[39] Al-Hiyali A, Ayoub A, Ju X, et al. The Impact of Orthognathic Surgery on Facial Expressions. J Oral Maxillofac Surg 2015; 73 : 2380-2390. doi:10.1016/j.joms.2015.05.008

[40] Kavin T, Jagadesan AGP, Venkataraman SS. Changes in quality of life and impact on patients' perception of esthetics after orthognathic surgery. J Pharm Bioallied Sci 2012; 4: S290S293. doi:10.4103/0975-7406.100276

[41] La Touche R. Craniofacial pain and disability inventory (CF-PDI) development and psychometric validation of a new que kopie. Pain Physician 2014: 95-108

[42] Bach M, Bach D, de Zwaan M, et al. Validierung der deutschen Version der 20-Item Toronto-Alexithymie-Skala bei Normalpersonen und psychiatrischen Patienten. [Validation of the German version of the 20 -item Toronto Alexithymia Scale in normal adults and psychiatric inpatients.]. PPmP Psychother Psychosom Med Psychol 1996; 46: 23-28

[43] Sullivan MJL, Bishop SR, Pivik J. The Pain Catastrophizing Scale: Development and Validation. Psychological Assessment 1996; 7: 524-532. doi:10.1037/1040-3590.7.4.524
[44] Nilges P, Essau C. Die Depressions-Angst-Stress-Skalen: Der DASS - ein Screeningverfahren nicht nur für Schmerzpatienten. Schmerz 2015; 29: 649-657. doi:10.1007| s00482-015-0019-z

[45] von Korff M, Dworkin SF, Le Resche L. Graded chronic pain status: an epidemiologic evaluation. Pain 1990; 40: 279-291. doi:10.1016/0304-3959(90)91125-3

Bibliografie

Der Schmerzpatient 2021; 4: 88-95

DOI $10.1055 / a-1345-2262$

ISSN 2512-6210

(C) 2021. Thieme. All rights reserved.

Georg Thieme Verlag KG, Rüdigerstraße 14,

70469 Stuttgart, Germany 